\title{
EFECTOS EN LOS USOS DEL SUELO DE LA DINÁMICA RECIENTE DEL MERCADO DEL RIOJA
}

\author{
T. LASANTA MARTÍNEZ \\ Instituto Pirenaico de Ecología (CSIC). \\ N. PASCUAL BELLIDO \\ Instituto de Estudios Riojanos (Gobierno de La Rioja)
}

\section{INTRODUCCIÓN.}

Desde hace dos décadas desciende en el mundo el consumo de vino de forma continuada en dos millones de hectólitros aproximadamente al año (RODRÍGUEZ DE LA CRUZ, 1994), lo que ha llevado al arranque de plantaciones en la Unión Europea y Argentina hasta muy recientemente, sin alcanzarse a veces los efectos buscados debido al aumento de los rendimientos (BARDAJÍ AZCÁRATE, 1994).

El descenso en el consumo no afecta, sin embargo, a todos los vinos. Los de baja calidad o sin etiqueta de especificidad experimentan un fuerte retroceso, mientras que los vinos de calidad con Denominación de Origen muestran una tendencia muy positiva, tanto en los países productores y consumidores tradicionales como en otros que se han incorporado recientemente al mercado de vino de calidad. El incremento de la renta de la población y la inclusión del vino en los hábitos gastronómico-culturales de las capas sociales de elevado poder adquisitivo son las razones fundamentales que explican el aumento del consumo de vino de calidad. Un factor a considerar también es la connotación del vino como producto sofisticado, lo que implica la apreciación por el consumidor de las características específicas de los distintos vinos. De ahí, que el reconocimiento de una marca o de una denominación de origen sea un factor destacado para mantenerse en el mercado, tratando las bodegas de adecuar su oferta a las exigencias del mercado.

En España, por ejemplo, los vinos con D.O. han pasado del 23,3\% al 35,5\% del total de las ventas durante la última década, según el ESTUDIO NIELSEN (1998) sobre el mercado de vinos en España elaborado para el Consejo Regulador de la Denominación de Origen Calificada Rioja (en adelante CRDOCa Rioja). Los vinos españoles se han beneficiado comercialmente, además, de la incorporación de España a la Unión Europea, lo que ha supuesto el desmantelamiento paulatino de barreras comerciales y la apertura de mercados poco permeables anteriormente a la importación de vinos españoles. De otra parte, la devaluación de la peseta a mediados de los noventa los ha hecho muy competitivos en los mercados europeos, incrementándose las exportaciones de 5,7 millones de hectólitros en 1980 a 7,1 millones de hectólitros en 1996 (PIQUERAS, 1997).

El Rioja participa muy activamente de la dinámica positiva del mercado de los

Polígonos, $\mathrm{n}^{\circ}$ 9, 1999, pp. 215-225. 
vinos de calidad, tanto por el volumen de sus ventas, que ha pasado de 103,7 millones de litros en 1990 a 222,3 millones de litros en 1998, como por el acceso a mercados cada vez más competitivos y exigentes en la calidad (MARTín y ALBISU, 1992; LASANTA, 1995). La evolución alcista del mercado implica cambios muy rápidos e importantes en el sector vitivinícola, tanto en la parte productora como en la elaboradora y comercializadora, que han sido apuntados por Lasanta (en prensa). En este trabajo se comentan algunos efectos en los usos del suelo debidos a la evolución del mercado del Rioja.

\section{EVOLUCIÓN RECIENTE DEL MERCADO DEL RIOJA.}

El CUADRO 1 informa sobre la comercialización del Rioja entre 1983 y 1998. Se observa que hasta 1990 hay una evolución en dientes de sierra, mientras que desde esta fecha la tendencia es claramente alcista, con un incremento del 114\% en los 9 últimos años, registrándose tanto en el mercado interior como en el exterior. Tan sólo durante los años 1995 y 1996 se produjeron ligeros retrocesos respecto al año 1994, aunque el volumen de ventas fue muy superior a los años precedentes. Conviene resaltar que la tendencia positiva del mercado se da a pesar del fuerte incremento de los precios (en 1988 el precio medio en el mercado exterior era de 331 ptas/litro y en 1998 de 586 ptas/litro) y de la competencia que establecen otras D.O. españolas, que han mejorado mucho la calidad y los canales de comercialización de sus vinos. Aún con todo, las ventas del Rioja representan alrededor del $40 \%$ del total de vinos con denominación vendidos en España (CUADRO 2) y el 17,5\% de las exportaciones de vino de mesa (PIQUERAS, 1997).

Cuadro 1: Evolución (1990 - 1998) de las ventas del Rioja en litros.

\begin{tabular}{rrrrr}
\hline Año & $\begin{array}{r}\text { Mercado Inter- } \\
\text { ior }\end{array}$ & Mercado Exterior & Total & Incremento \\
\hline 1983 & 76.804 .202 & 31.046 .488 & 107.850 .692 & $-0,9$ \\
1984 & 68.240 .235 & 28.471 .311 & 96.711 .669 & $-11,4$ \\
1985 & 67.743 .375 & 28.633 .625 & 96.377 .000 & $-0,4$ \\
\hline 1986 & 74.651 .578 & 29.902 .122 & 104.553 .700 & 8,4 \\
1987 & 77.442 .665 & 35.665 .735 & 113.108 .400 & 8,2 \\
1988 & 82.894 .300 & 34.301 .700 & 117.196 .000 & 3,6 \\
1989 & 77.784 .543 & 26.631 .578 & 104.416 .121 & $-11,0$ \\
1990 & 77.752 .631 & 26.029 .946 & 103.782 .577 & $-0,61$ \\
\hline 1991 & 99.841 .642 & 29.137 .809 & 128.979 .451 & 24,28 \\
1992 & 114.478 .913 & 34.362 .372 & 148.841 .285 & 15,4 \\
1993 & 120.199 .494 & 38.722 .771 & 158.922 .265 & 6,77 \\
1994 & 139.918 .321 & 56.790 .139 & 196.708 .460 & 23,78 \\
1995 & 125.953 .908 & 59.116 .887 & 185.070 .795 & $-5,92$ \\
\hline 1996 & 119.444 .519 & 58.846 .727 & 178.291 .246 & $-3,66$ \\
1997 & 137.458 .184 & 67.781 .317 & 205.239 .501 & 15,11 \\
1998 & 150.699 .111 & 71.550 .035 & 222.249 .146 & 8,16 \\
\hline F & 1030
\end{tabular}

FUENTE: CRDOCa Rioja. Elaboración propia 
Las ventas del Rioja en 1988 fueron de 26.275 millones de pesetas, mientras que en 1998 ascendieron a 118.669 millones de pesetas (de los que el 64,6\% aproximadamente corresponde al mercado interior y el resto al exterior).

La demanda creciente de vino de Rioja ha repercutido en el precio de la uva, que ha experimentado un notable ascenso, especialmente durante los últimos años. En Logroño, que puede ser considerado como un municipio de precios medios en la DOCa Rioja, se pagó a 5 ptas $/ \mathrm{kg}$ en 1970, a 23 ptas $/ \mathrm{kg}$ en 1980 y a 100 ptas/kg en 1988; en los años siguientes descendió, aunque nunca por debajo de 45 $\mathrm{ptas} / \mathrm{kg}$. Desde 1990 presenta una tendencia muy positiva, ascendiendo de forma prácticamente constante: en 1990 se pagó a $45 \mathrm{ptas} / \mathrm{kg}$, en 1994 a $70 \mathrm{ptas} / \mathrm{kg}$ y en 1998 a 200 ptas/kg. LASANTA (en prensa) aporta información más detallada.

Cuadro 2: Participación (en \%) de las D.O. españolas en el mercado nacional de alimentación y hostelería.

\begin{tabular}{lrrrrr}
\hline & $\mathbf{1 9 8 8}$ & $\mathbf{1 9 9 3}$ & $\mathbf{1 9 9 5}$ & $\mathbf{1 9 9 7}$ & $\mathbf{1 9 9 8}$ \\
\hline Rioja & 39,9 & 41,8 & 41,3 & 40,0 & 39,1 \\
Navarra & 6,5 & 6,7 & 7,8 & 7,0 & 7,3 \\
Valdepeñas & 15,5 & 11,5 & 10,9 & 11 & 10,3 \\
Jumilla & 4,3 & 7,2 & 6,3 & 5,8 & 5,5 \\
Cariñena & - & 3,0 & 3,8 & 3,6 & 1,9 \\
\hline Penedés & 6,9 & 5,9 & 6,8 & 8 & 8,1 \\
Mancha & 6,5 & 4,4 & 3,3 & 3,1 & 3,8 \\
R. del Duero & - & 2,9 & 3,6 & 4,5 & 3,9 \\
Rueda & - & 1,6 & 2,6 & 1,9 & 2,9 \\
Ribeiro & 6,5 & 5,5 & 3,7 & 4,0 & 4,0 \\
\hline Rías Bajas & - & - & 1 & 1,4 & 1,6 \\
Somontano & - & - & - & 0,8 & 1,1 \\
Valencia & - & - & - & 2 & 1,5 \\
Otras & 14,2 & 9,4 & 9,5 & 8,0 & 8,6 \\
\hline Mercado total (mill. litros) & 143 & $S D$ & 201 & 212,8 & 233 \\
\hline FU
\end{tabular}

FUENTE: CRDOCa Rioja. Elaboración propia.

El incremento del precio de la uva junto a la mejora de la productividad, como veremos posteriormente, ha hecho muy rentable el cultivo del viñedo. En un estudio reciente ("Costes de producción de cultivos agrícolas en La Rioja") de la Consejería de Agricultura, Ganadería y Desarrollo Rural del Gobierno de La Rioja se le calcula un beneficio medio de 1.297.024 ptas/Ha con la producción media y el precio medio de 1998, lo que le hace ser el cultivo más rentable de los que se dan en la región (CUADRO 3). El mencionado estudio señala también que por encima de las $65 \mathrm{ptas} / \mathrm{kg}$ se obtienen beneficios con producciones de $5.000 \mathrm{Kg} / \mathrm{Ha}$, siendo tanto este precio como la producción muy inferior es a la media de los últimos años. 
Cuadro 3: Beneficios por hectárea de varios cultivos representativos de La Rioja (1988).

\begin{tabular}{lrr}
\hline \multicolumn{1}{c}{ Cultivo } & Pts/Ha & Pts/Kg \\
\hline Trigo secano & 36.868 & 7,374 \\
Cebada secano & 14.715 & 3,270 \\
\hline Patata media estación & 209.608 & 3,88 \\
Remolacha regadío & 161.038 & 2,301 \\
\hline Guisante verde regadío & 68.776 & 9,552 \\
Alubia verde regadío & 380.698 & 17,30 \\
\hline Coliflor regadío & 380.698 & 17,30 \\
Viñedo secano & 1.297 .024 & 158,174 \\
\hline
\end{tabular}

\section{CAMBIOS EN LOS USOS DEL SUELO.}

PASCUAL y CABRERIZO (1995) resumen las exigencias ambientales de la vid y señalan los límites más apropiados para obtener un producto de calidad. Esquematizando mucho diremos que se dan cuando el viñedo se localiza en suelos profundos y pedregosos con un porcentaje de caliza (los mejores viñedos aparecen en suelos con caliza activa del $15 \%$ aproximadamente y entre el 2 y el $5 \%$ de caliza activa, según RUIZ HERNÁNDEZ, 1988), lo que en La Rioja aparece fundamentalmente en glacis altos y terrazas altas. Las precipitaciones deben estar entre 400$600 \mathrm{~mm} / a$ ño, con un mínimo de insolación de 1.500-1.600 horas/año, de las que al menos 1.200 horas deben registrarse durante el periodo vegetativo, especialmente en las fechas de maduración (final del verano, principio de otoño).

En relación con los requisitos señalados, La Rioja Alta y La Rioja Alavesa constituyen los espacios más idóneos para producir vinos de calidad dentro de la región, al combinarse el dominio de suelos pardo-calizos con unas buenas condiciones climáticas. La influencia atlántica aporta precipitaciones abundantes y suaves temperaturas, mientras que el efecto Foëhn, como consecuencia de su localización a sotavento de la Cordillera Cantábrica, incrementa la integral térmica; estos condicionantes climáticos no se producen en La Rioja Baja (de influencia mediterránea), donde los contrates térmicos rompen el equilibrio que necesita la vid para ofrecer un buen producto, y donde las precipitaciones (por debajo frecuentemente de los $400 \mathrm{~mm} / \mathrm{año}$ ) resultan relativamente escasas (ANDRADAS RODRÍGUEZ, 1991).

Aún con todo, dentro de cada comarca y de cada municipio existen áreas más y menos favorables. Así, los glacis altos y terrazas altas son muy adecuados para el viñedo al recibir alta insolación y escapar de heladas causadas por inversiones térmicas. Además, tienen suelos sueltos y muy pedregosos, lo que favorece la infiltración del agua e incrementa la integral térmica, lo que resulta muy interesante en la fase final de maduración.

MANZANARES (1988) estudió las áreas de localización del viñedo, mediante fotografía aérea, en 1956 y 1978. La primera fecha puede servir de referencia para conocer la distribución del viñedo al final del sistema tradicional, cuando el viñe- 
do cumplía básicamente una función de autoabastecimiento y en segundo lugar comercial, pero sin entrar en competencia con otros usos agrícolas del suelo que tambén eran necesarios para la autosubsistencia. Ello daba lugar a una localización que no siempre era la más idónea, ya que a veces se debían utilizar espacios marginales cercanos incluso al límite ecológico para el cultivo de la vid, como ocurría en la mayoría de los pueblos de montaña. En estas condiciones la vid ocupaba glacis altos y bajos, pero también conos de deyección muy pedregosos y barrancos en $\mathrm{V}$, muy encajados pero relativamente abrigados.

La segunda fecha puede reflejar una localización más en función del mercado que del autoabastecimiento, lo que llevaría a ocupar los espacios más idóneos, que en el caso de la vid tienen que ver con la obtención de la mejor calidad. En esta fecha, MANZANARES (1988) señala que el viñedo se sitúa en glacis altos y en laderas con pendientes relativamente fuertes, donde ni los cereales pueden competir, mientras que ha desaparecido de glacis y terrazas bajas, siendo sustituidos por los cereales de regadío, de los valles en $\mathrm{V}$ y de laderas muy pendientes no mecanizables, dejando paso a campos abandonados, a la vez que reduce mucho su superficie de los conos de deyección.

Durante los últimos años la localización nuevamente vuelve a cambiar como consecuencia de los elevados beneficios ofrecidos por el viñedo, lo que le ha hecho el cultivo más atractivo para los agricultores, capaz de desplazar o sustituir a cualquier otro. Espacialmente se refleja en su expansión superficial. El CUADRO 4 anota la evolución de la superficie de viña en la DoCa Rioja desde 1983. Conviene señalar, inicialmente, que la superficie cultivada es mayor, ya que se han plantado viñedos sin derechos de plantación, por lo que no están inscritos en la DOCa Rioja. BARCO (1991) calculó que en 1990 había 5.834 Ha de plantaciones ilegales, lo que suponía incrementar la superficie de aquel año en el 10,2\%. En los últimos años posiblemente ha aumentado el porcentaje de viñedo no inscrito, lo que se deduce de la elevada cotización de los derechos de plantación (alrededor de 2 millones de ptas/Ha) y del creciente número de solicitudes no autorizadas de nuevas plantaciones. Aún con todo, el CUADRO 4 pone claramente de manifiesto que en las tres provincias que integran la DoCa Rioja la superficie de viñedo ha aumentado de forma muy considerable: en La Rioja el 34,7\%, en Alava el 42,4\% y en Navarra el 67,7\%. Para el conjunto de la denominación el aumento de superficie ha sido de $14.889 \mathrm{Ha}$, lo que equivale a un incremento del $38,8 \%$ en 15 años, aunque incluyendo el viñedo no legalizado superará el 50\% muy probablemente.

La ampliación superficial del viñedo (ver FIGURA 1 para la provincia de La Rioja) ha tenido lugar, fundamentalmente, en los municipios más viticultores, donde el viñedo constituye prácticamente un monocultivo, como ocurre, por ejemplo, en Abalos, Briñas, Cenicero, Fuenmayor, San Asensio, San Vicente de la Sonsierra, Torremontalvo, Uruñuela, Laguardia, Elciego, Labastida, Lanciego y El Villar de Alava. En otros la expansión del viñedo ha sido también espectacular, aunque siempre limitada por el control y las restricciones para efectuar nuevas plantaciones, como en algunos municipios de La Rioja Baja (Alfaro, Ausejo, Quel, El Villar de Arnedo), que reúnen condiciones climáticas y edáficas menos adecuadas que La Rioja Alta y La Rioja Alavesa. 

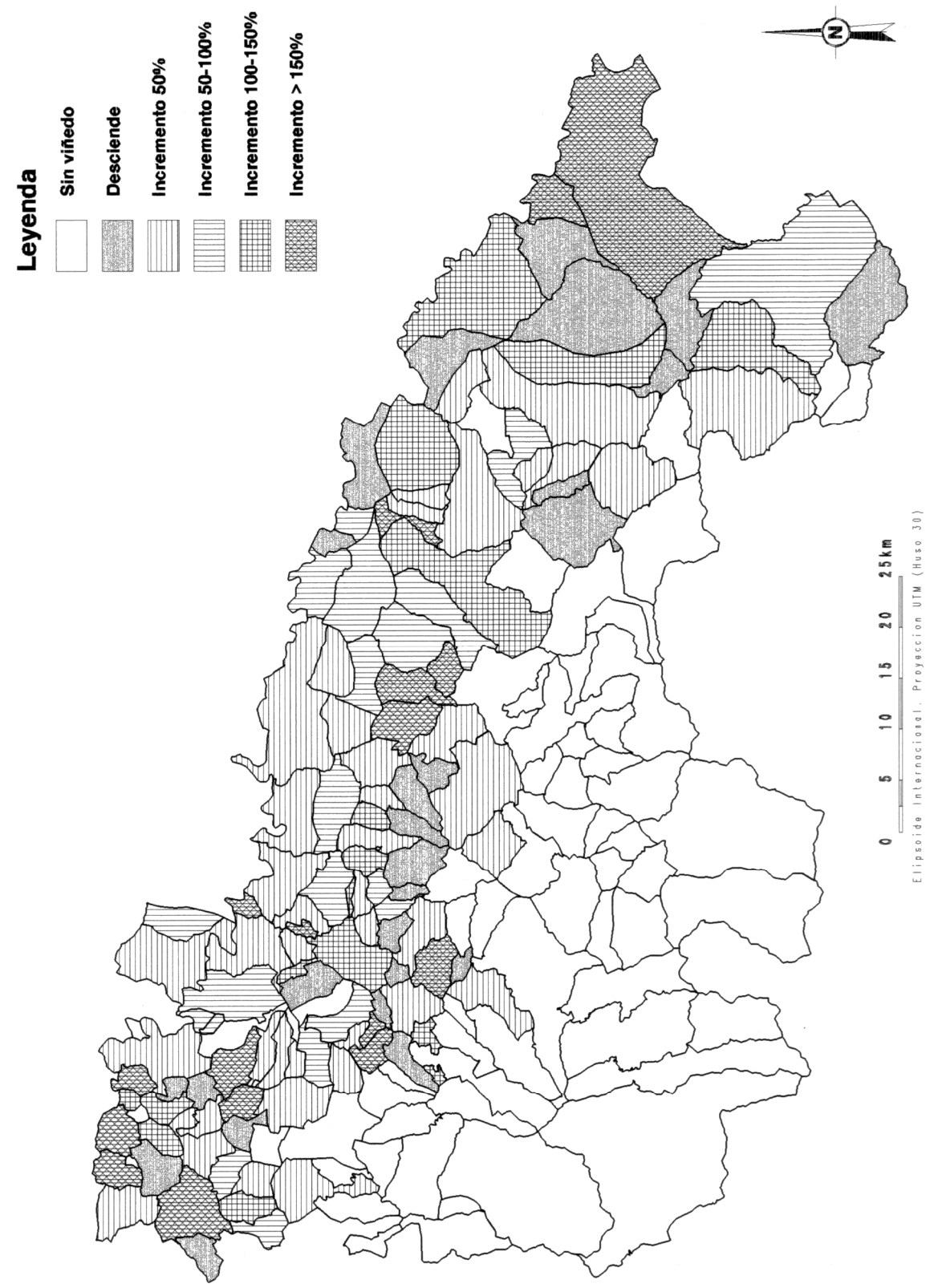
Cuadro 4: Evolución del viñedo inscrito en la DOCa Rioja en Has (19831998).

\begin{tabular}{rrrrr}
\hline Año & La Rioja & Álava & Navarra & Total \\
\hline 1983 & 27.395 & 8.039 & 2.915 & 38.349 \\
1984 & 28.524 & 8.635 & 3.050 & 40.209 \\
1985 & 29.736 & 8.968 & 3.036 & 41.740 \\
\hline 1986 & 30.519 & 9.436 & 3.120 & 43.076 \\
1987 & 31.850 & 9.727 & 3.092 & 44.669 \\
1988 & 31.640 & 9.823 & 3.631 & 45.094 \\
1989 & 30.814 & 9.866 & 3.880 & 44.560 \\
1990 & 32.231 & 10.261 & 4.480 & 46.972 \\
\hline 1991 & 32.203 & 10.221 & 4.751 & 47.175 \\
1992 & 33.240 & 10.293 & 4.912 & 48.445 \\
1993 & 34.118 & 10.498 & 5.078 & 49.649 \\
1994 & 35.100 & 10.767 & 4.986 & 50.853 \\
1995 & 34.909 & 10.828 & 4.898 & 50.635 \\
\hline 1996 & 35.169 & 10.815 & 4.789 & 50.733 \\
1997 & 36.406 & 11.157 & 4.701 & 52.264 \\
1998 & 36.903 & 11.445 & 4.890 & 53.238 \\
\hline
\end{tabular}

FUENTE: CRDOCa Rioja

Hay que señalar, también, que incluso en las comarcas con buenas condiciones edáficas y climáticas la expansión reciente del viñedo no siempre se ha realizado en las tierras más apropiadas, utilizando cualquier campo sin tener muy en cuenta sus condiciones edáficas y topográficas. Unas de la áreas preferidas para las nuevas plantaciones de viñedo son las parcelas de regadío con suelo muy fértil. Según la información incluida en las Memorias Anuales de Estadística Agraria de la Consejería de Agricultura, Ganadería y Desarrollo Rural del Gobierno de La Riojase plantaron 4.279 Ha de viñedo, de las que 1.340 lo fueron en regadío y 2.939 en secano entre 1987 y 1996. En 1987 había 1.039 Ha en regadío, lo que significa un incremento del 129\% del viñedo en regadío en 10 años. La misma fuente indica respecto al viñedo que aún no produce, es decir el de nueva plantación, que en regadío había 63 Ha en 1994, 65 Ha en 1995, 213 Ha en 1996, 244 Ha en 1997 y $358 \mathrm{Ha}$ en 1998, lo que demuestra la progresiva preferencia por el regadío, desplazando a cultivos muy exigentes en mano de obra como las hortalizas y los frutales.

La productividad se ha ido incrementado progresivamente: en el periodo 197983 el rendimiento medio era de $4.428 \mathrm{Kg} / \mathrm{Ha}$, en el periodo $1984-1988$ de 4.824 $\mathrm{Kg} / \mathrm{Ha}$, en $1989-1993$ de $5.160 \mathrm{Kg} / \mathrm{Ha}$ y de $6770 \mathrm{~kg} / \mathrm{Ha}$ en $1994-1998$. El aumento de los rendimientos se explica por muy diferentes factores, entre los que destacan el rejuvenecimiento de las plantaciones por la incorporación de nuevos viñe- 
dos y la sustitución de viñas viejas por nuevas (Barco, 1990), el uso de suelos más fértiles, el empleo de regadío y la mayor incorporación de "inputs" (fertilizantes, productos fitosanitarios y sobre todo mayor dedicación de trabajo). En relación con el último aspecto hay que señalar que la Consejería de Agricultura, Ganadería y Desarrollo Rural del Gobierno de La Rioja calcula una inversión de 155 horas/Ha/año, mientras que a principios de los ochenta era de 110 horas/Ha/año (Lasanta y Frias, 1984), pese al incremento de la mecanización de muchas labores. La mayor dedicación del viñedo va en detrimento de otros cultivos, que son progresivamente marginados. Aunque no se debe establecer una relación directa entre dedicación al viñedo y evolución superficial de otros cultivos, sí que es cierto que los más exigentes en mano de obra (hortalizas, patatas, frutales de regadío) registran las mayores pérdidas de superfice. Entre 1990 y 1998 las hortalizas disminuyen el $24 \%$ de superficie (de $15.132 \mathrm{Ha}$ a $11.541 \mathrm{Ha}$ ), siendo sustituidas además las más exigentes en mano de obra (pepinillo, tomate, guindilla) por otras totalmente mecanizadas (guisante y judia verde). En el mismo sentido, los frutales pierden el 13\% de su superficie (de 7.046 a $6.138 \mathrm{Ha}$ ) y las patatas el $56 \%$ (de 7.639 a $3.361 \mathrm{Ha}$ ), teniendo lugar los descensos más importantes en algunos de los municipios que plantan más viñedo.

El viñedo constituye, pues, durante los últimos años un fuerte competidor por el uso del suelo de los espacios más fértiles, pero también por la utilización de la mano de obra. Desde una perspectiva socioeconómica, el viñedo se ha convertido en el cultivo fundamental en el territorio que comprende la DoCa Rioja, lo que ha llevado, por una parte, a la sustitución de cultivos y, por otra, a la extensificación del resto de los usos agrícolas del suelo al haberse intensificado el viñedo, que absorbe la mayor parte de la mano de obra.

\section{DISCUSIÓN Y CONCLUSIONES.}

Uno de los cambios más importantes registrados por el sector primario es el paso de abastecer un mercado local a hacerlo de un mercado de más amplio radio. El desarrollo de los transportes, de los canales de comercialización y de la tecnología del envasado ha hecho que la agricultura reciente compita en un mercado mundial. El acceso al mercado se efectúa unas veces mediante la obtención de productos más baratos que en otras áreas productoras, y otras con el abastecimiento de productos muy específicos, con etiqueta diferenciadora (Denominación de Origen), que suele vincularse a los condicionantes físicos de un territorio determinado y/o a la cultura de elaboración del lugar de procedencia. El vino se incluye en la segunda estrategia comercial. No hay que olvidar que el vino de calidad exige que el viñedo tenga unas condiciones ambientales muy concretas en clima y suelo, lo que reduce mucho el área geográfica de los viñedos. Además, el vino requiere un proceso de elaboración minucioso, donde son determinantes las condiciones de almacenamiento y los conocimientos sobre la manipulación. Por último, el vino es un producto no perecedero; el paso del tiempo puede mejorar la calidad, elevar el precio y consecuentemente incrementar el valor añadido. Este hecho le permite acceder a cualquier mercado, por muy lejano que se encuentre.

La liberalización del mercado, como consecuencia de los acuerdos GATT, y el 
ingreso de España en la UE, con el descenso de la fiscalidad y el incremento del consumo de vinos de calidad, ha favorecido el aumento de las ventas de los vinos tranquilos y espumosos españoles, entre los que participa de forma destacada el Rioja (PIQUERAS, 1997). Es necesario recordar, que con anterioridad a la entrada de España en la UE, el viñedo era un sector relativamente marginal, con una producción de vino muy abundante en relación con la demanda interior y con el escaso volumen de exportación (la mayor parte a granel), por lo que era incapaz de competir en mercados abiertos, en los que la calidad era un aspecto muy valorado.

Sin embargo, durante la última década las ventas del Rioja se han duplicado, a la vez que el valor de ellas se ha multiplicado por cuatro: en 1988 ascendía a 26.275 millones de pesetas, mientras que en 1998 superaba ligeramente los 118.000 millones de pesetas. El aumento espectacular de las ventas se ha apoyado en la expansión superficial del viñedo (entre 1983 y 1998 se plantaron al menos $15.000 \mathrm{Ha}$, lo que supuso incrementar la extensión en el $38,8 \%$ respecto a la primera fecha) y en la mejora de la productividad (los rendimientos medios son el 53\% superiores en 1994-98 que en el periodo 1979-83).

La expansión del viñedo muestra cierto grado de concentración, en la medida en que los municipios más vitivinícolas de La Rioja Alta y La Rioja Alavesa son los que han plantado más viñas recientemente, lo que ha llevado a la ocupación de tierras poco adecuadas para producir vino de calidad. La vid se había acomodado a lo largo del tiempo a su ecotopo más favorable, sin entrar a competir por los suelos más fértiles que quedaban para cultivos más exigentes y con menor capacidad de adaptación a condiciones topográficas difíciles. Por otro lado, en La Rioja Baja también se ha expandido, a pesar de que las condiciones climáticas favorecen vino de mayor grado alcohólico que se aleja del Rioja tradicional.

Desde una perspectiva geoecológica la ampliación superficial reciente del viñedo implica un cambio radical respecto a la ocupación de tierras en otras épocas de expansión, que se había realizado mediante la roturación de tierras marginales, proceso precedido con frecuencia de la deforestación de encinares y carrascales. GARCÍA-RUIZ y ARNÁEZ (1987) lo ponen de manifiesto en el municipio de Cenicero, donde desde mediados del siglo XVIII se desmontaron bosques de encina para plantar viñedos. En el mismo sentido, MANZANARES (1987) indica que el encinar de la Dehesa de Navarrete (glacis pliocuaternario, nivel VI del Ebro, según GONZALO MORENO, 1981) fue roturado para el cultivo de viñedos.

Se ha señalado, también, que una parte del nuevo viñedo se localiza en campos de regadío, lo que tiene sus ventajas e inconvenientes. Entre las primeras hay que destacar la mayor producción, especialmente en años secos. Entre los inconvenientes se incluyen el adelanto del ciclo vegetativo, con el consiguiente riesgo de sufrir heladas tardías y el desarrollo de mayor número de plagas, así como la posibilidad de alcanzar graduaciones alcohólicas más bajas. De ahí, que el riego sea una técnica muy problemática porque su expansión indiscriminada podría generar un efecto negativo de cara a la comercialización, por el aumento de la producción y por el posible descenso de la calidad.

En definitiva, la tendencia positiva del mercado del Rioja durante los últimos años ha impulsado importantes cambios en el sector vitivinícola (LASANTA, en prensa). En este trabajo se han señalado algunas modificaciones en los usos del 
suelo y en la gestión de los viñedos, insistiendo en que una proporción importante de las nuevas plantaciones se localizan en áreas que tradicionalmente no han sido consideradas como las más adecuadas. Asimismo, se ha puesto de relieve el fuerte incremento de la productividad en relación con la renovación del viñedo, la intensificación del laboreo y el mayor añadido de "inputs" (LASANTA, en prensa). Una vez más se demuestra el poder creciente del mercado en la distribución de los usos del suelo. Tradicionalmente, el agricultor interpretaba la diversidad ambiental y aprovechaba al máximo los flujos energéticos, dando lugar a unos usos del suelo muy controlados por factores topográficos, geomorfógicos y edáficos. La penetración en mercados amplios y potentes introduce cambios en la distribución espacial de los cultivos, favoreciendo la expansión de los más dinámicos y rentables económicamente, sin tener muy en cuenta sus exigencias ambientales, que son superadas por la incorporación de productos químicos en la fase de cultivo o en las de transformación y elaboración.

Se plantea la duda de si estos rasgos de la evolución reciente pueden garantizar el mantenimiento de la calidad que ha dado prestigio al Rioja, o si ésta cada vez estará más influida por la fase de elaboración, lo que llevaría a cierta estandarización y facilitaría la imitación de los vinos de Rioja por otras zonas productoras. Desde esta perspectiva, parece más adecuada una estrategia muy exigente en la localización del viñedo, de forma que el perfil característico de los vinos incluidos en la Denominación esté condicionado fuertemente por los factores ambientales.

\section{REFERENCIAS BIBLIOGRAFICAS.}

- ANDRADES RODRÍGUEZ, A. (1991): Influencias climáticas sobre el proceso de maduración del fruto de vitis vivifera. Diferenciación varietal. Gobierno de La Rioja, Serie Estudios, 20.

- BARCO, E. (1991): Análisis de un sector: El Rioja (1983-1990). Gobierno de La Rioja. Serie Estudios, 23: 126 pp. Logroño.

- BARDAJ́ AZCÁRATE, I. (1994): «Modificación de la oferta internacional del vino». El Campo, 130: 197-208.

- GARCÍA-RUíz, J.M. y ARNÁEZ, J. (1987): «El medio natural». En: Cenicero histórico. Ayuntamiento de Cenicero - Comunidad Autónoma de La Rioja: 1156, Logroño.

- GOBIERNO DE LA RIOJA (1999): Coste de producción de cultivos agrícolas en La Rioja. Consejería de Agricultura, Ganadería y Desarrollo Rural: 109 pp., Logroño.

- GonZalo Moreno, A.N. (1981): El relieve de La Rioja. Análisis de Geomorfología Estructural. Instituto de Estudios Riojanos, 2 vols., Logroño.

- LASANTA, T. (en prensa): «Evolución reciente del mercado del Rioja y cambios en el sector vitininícola». Berceo.

- LASANTA, T. (1995): «La exportación del Rioja en el contexto del mercado mundial del vino». Berceo, 129: 55-74.

- LASANTA, T. y FRÍAS, R. (1984): «Aspectos condicionantes de la morfología en la agricultura periurbana: evolución de los usos del suelo y de la gestión en la agricultura próxima a Logroño». Berceo (Ciencias), 2: 115-133. 
- LASANTA, T. (en prensa): «Evolución reciente del mercado del Rioja y cambios en el sector vitivinícola». Berceo.

- MANZANARES SiERRA, C. (1987): «El impacto de las transformaciones del medio rural en la utilización de los bosques: los encinares de La Rioja». Estudios Geográficos, 189: 619-637.

- MANZANARES SIERRA, C. (1988): «Influencia de los factores topogeomorfológicos en la distribución y evolución de cultivos en el valle del Ebro riojano». Berceo, 118: 43-51.

- MARTín, D. y ALBISU, L.M. (1992): El mercado del vino en la Denominación de Origen Rioja. MAPA: 242 pp., Madrid.

- NIELSEN (1993-1998): En: Memoria del CR.DOCa Rioja. Logroño, informes anuales.

- PASCUAL, N. y CABRERIZO, A. (1995): «Distribución espacial del viñedo de Rioja en relación con los condicionantes ambientales». Berceo, 129: 75-95.

- PIQUERAS, J. (1997): «La exportación del vino en España. Los efectos de la integración en la Unión Europea». Cuadernos de Geografía, 61: 117-143.

- RODRÍGUEZ DE LA CRUZ, G. (1994): «Reflexiones sobre la Nueva OCM del vino». El Campo, 130: 259-275.

- RUIZ HeRNÁNDEZ, M. (1988): «El vino de Rioja». El Campo, 110: 55-66. 